\title{
Estaca de Lauraceae em contexto funerário (sítio Jaboticabeira II, Santa Catarina, Brasil)
}

\author{
Gina Faraco Bianchini* \\ Rita Scheel-Ybert** \\ Maria Dulce Gaspar***
}

\begin{abstract}
BIANCHINI, G.F.; SCHEEL-YBERT, R.; GASPAR, M.D. Estaca de Lauraceae em contexto funerário (sítio Jaboticabeira II, Santa Catarina, Brasil). Revista do Museu de Arqueologia e Etnologia, São Paulo, 17: 223-229, 2007.
\end{abstract}

Resumo: A antracologia tem contribuído com numerosas informações relativas à utilização de recursos vegetais por grupos sambaquieiros. A análise antracológica de uma estaca carbonizada associada a uma estrutura funerária do sítio Jabuticabeira-II permitiu a identificação de uma Lauraceae (Ocotea sp.) e levantou hipóteses sobre a prática de coleta seletiva de recursos vegetais. Esta seleção poderia ser orientada por aspectos funcionais associados às características da madeira e/ou por aspectos simbólicos associados ao domínio cosmológico do grupo.

Palavras-chave: Antracologia - Paleoetnobotânica - Sambaquis Ritual funerário.

\section{Introdução}

s recursos vegetais desempenham importante papel para diversas culturas humanas desde seus primórdios (Prance 2005), servindo como fonte variada e abundante de suprimentos que pode ser empregada em um universo infinito de aplica-

$\left({ }^{*}\right)$ Museu Nacional, Universidade Federal do Rio de Janeiro-UFRJ. Mestrado em Arqueologia. ginabianchini@hotmail.com

${ }^{(* *)}$ Museu Nacional, Universidade Federal do Rio de JaneiroUFRJ. Departamento de Geologia e Paleontologia. Pesquisadora do CNPq.scheelybert@mn.ufrj.br $\left.{ }^{* * *}\right)$ Museu Nacional, Universidade Federal do Rio de Janeiro-UFRJ. Departamento de Antropologia. Pesquisadora do CNPq.madugaspar@terra.com.br ções e domínios como alimentação, construção, fabricação de artefatos, transformação de matérias-primas (combustível) e ritual.

Tais aplicações exigem um profundo conhecimento das propriedades e potenciais de determinadas espécies, resultando numa relação íntima entre humanos e plantas que é condição necessária tanto para a coleta e extração quanto para o processamento de vegetais. Estudos etnográficos mostram que é freqüente a especialização de conhecimento em diferentes segmentos de um mesmo grupo social (homens/mulheres, jovens/adultos, líderes/não líderes). Em comunidades caiçaras do litoral sul de São Paulo, por exemplo, o conjunto de atividades praticadas e o grau de familiaridade com cada ambiente conduzem a um conhecimento diferenciado entre homens (vegetação mais distante da casa) e 
mulheres (recursos próximos à base familiar) (Hanazaki 2001).

Por outro lado, a eleição de determinadas espécies para um uso específico está freqüentemente associada não somente às suas propriedades físico-químicas, mas sim a fatores simbólicos e/ou cosmológicos que ultrapassam princípios funcionais. Aspectos cognitivos, enquanto domínios inerentes aos seres humanos, influenciaram fortemente o modo pelo qual plantas foram e/ou deixaram de ser utilizadas e/ou incorporadas ao cotidiano de diversas sociedades.

Mas tratar destas questões em contexto arqueológico é tarefa difícil por uma série de razões. Vestígios vegetais compõem uma parcela ínfima da maior parte dos registros materiais, o que se deve essencialmente à ação de processos tafonômicos. Além disso, a dificuldade inerente ao trabalho de identificação botânica faz com que poucos pesquisadores se lancem neste desafio, com isso reduzindo ainda mais as informações disponíveis sobre o tema. A raridade de vestígios resulta numa impressão errônea de que a relação com o universo vegetal seria secundária em relação a atividades de caça e pesca. No entanto, os vegetais fizeram parte do universo humano no passado de modo muito intenso, ao contrário do que vem sendo geralmente afirmado em reconstituições arqueológicas.

No Brasil, até o final da década de 90, os vestígios vegetais eram muito pouco considerados junto à pesquisa arqueológica. A possibilidade de uso dos recursos vegetais no cotidiano de grupos pré-históricos era deduzida indiretamente de inferências baseadas em artefatos líticos funcionalmente associados ao processamento de vegetais. A partir de meados desta década, a obtenção de um maior número de evidências diretas destes recursos foi possibilitada pelo investimento em técnicas de resgate, em escavações por decapagens e pelo uso de flotação (Scheel-Ybert 2004). A partir da divulgação da antracologia, os carvões arqueológicos deixaram de ser simplesmente uma fonte de material para datação radiocarbônica, passando também a ilustrar a utilização dos vegetais na cultura sambaquieira, especialmente com relação à reconstituição paleoambiental, à economia de combustível e à dieta (ScheelYbert 1999, 2001, 2003; Scheel-Ybert et al. 2003). No entanto, ainda há uma série de questões com as quais a análise dos vestígios vegetais pode contribuir, desde que sejam seguidos procedimentos de campo especialmente estabelecidos para a recuperação destes vestígios (Scheel-Ybert et al. 2005).

Este artigo se propõe a discutir alguns aspectos da utilização de madeira por um grupo sambaquieiro, partindo da análise antracológica de fragmentos de carvão resgatados de um buraco de estaca associada a uma estrutura funerária do sítio Jabuticabeira-II. Existem evidências de um forte investimento no cuidado com os mortos dentro da soiedade sambaquieira (Klökler 2001; Gaspar 2000; Fish 2000), de modo que as estruturas associadas à parafernália ritualística certamente teriam um alto valor simbólico que deve ser considerado.

\section{Material e métodos}

O sítio Jabuticabeira-II localiza-se no município de Jaguaruna, litoral sul do estado de Santa Catarina, georreferenciado pelas coordenadas 699489/6835694 UTM. Apresenta uma superfície de $400 \mathrm{~m}$ x $150 \mathrm{~m}$, elevando-se a cerca de $8 \mathrm{~m}$ de altura. $\mathrm{O}$ sítio é formado por uma série de camadas alternadas que refletem níveis de atividade de ocupação (camadas funerárias) e níveis de construção (camadas de conchas e sedimento arenoso). As camadas funerárias apresentam um grande número de artefatos, restos de fauna, fragmentos de carvão e estruturas de combustão. Nelas ocorrem também diversos sepultamentos, ao redor dos quais se distribuem numerosos buracos de estacas de forma circular ou semicircular (Fig. 1). Estruturas similares são recorrentes no registro arqueológico de diferentes grupos, sendo geralmente associadas a feições funerárias ou de moradia (Dias Jr. 1980; Gaspar 2000; Schmitz 2006; Prous 1992). No caso do sítio Jabuticabeira-II, as evidências têm demonstrado se tratar de estruturas associadas à parafernália ritual funerária (Fish 2000). 


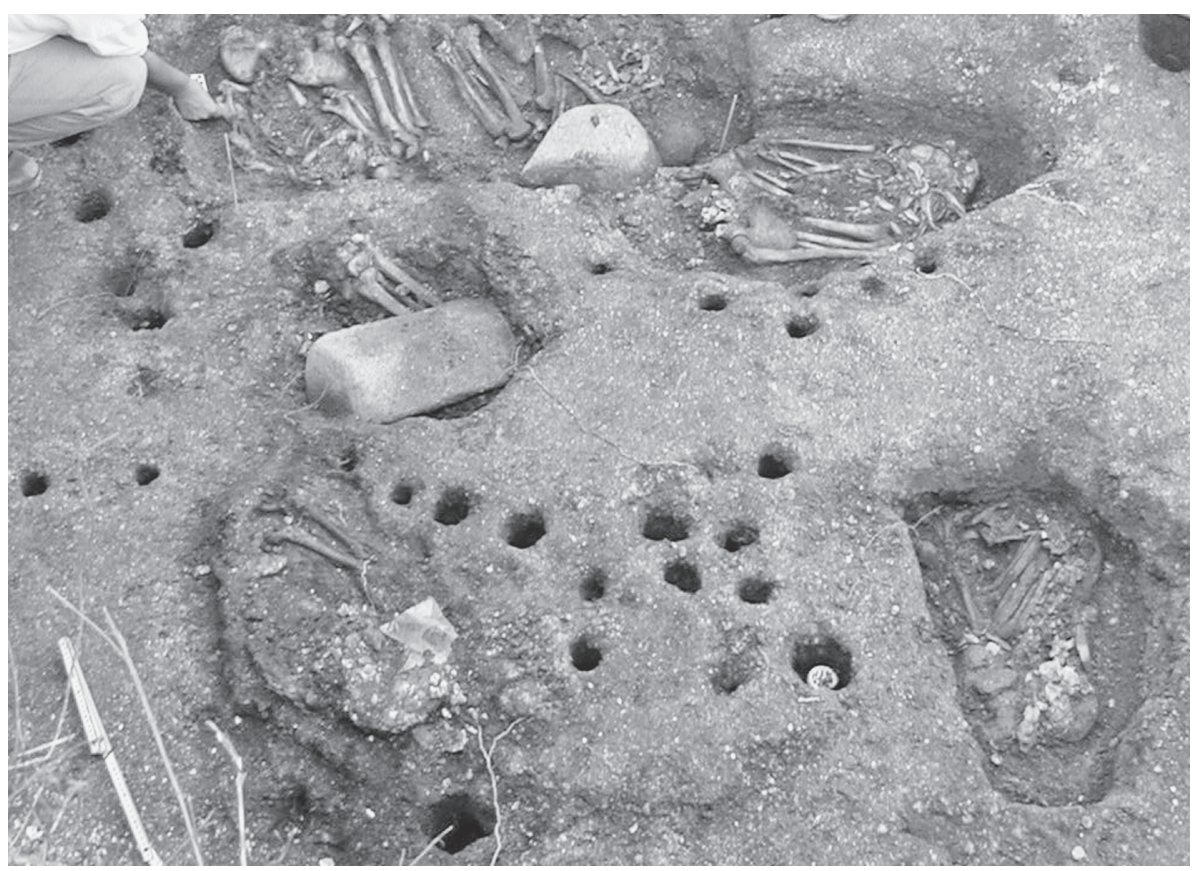

Fig. 1. Aspecto da escavação no Sambaqui Jabuticabeira-II, ilustrando uma área funerária com sepultamentos e buracos de estaca.

O material analisado foi resgatado de um buraco de estaca associado ao sepultamento 12 do Locus 1 (perfil 1.25, feição 1.25.4). Esta estrutura foi descrita como uma superfície com buracos de estacas, areia cinza e cinzas (Simões 2007).

No total, 66 fragmentos de carvão foram coletados. Todo o material foi analisado em microscopia de luz refletida com campo claro e campo escuro, após quebra manual dos fragmentos segundo os três planos fundamentais da madeira (transversal, longitudinal tangencial e longitudinal radial). As descrições morfométricas e qualitativas da anatomia do lenho seguiram normas estabelecidas internacionalmente (IAWA Committee 1989). A determinação taxonômica foi feita com o auxílio do banco de dados "Atlas Brasil" (Scheel-Ybert et al. 2006), e da comparação com amostras atuais da antracoteca do Museu Nacional (UFRJ) e com dados publicados na literatura (cf. Scheel-Ybert 2004; Scheel-Ybert et al. 2006).

\section{Resultados}

Todos os fragmentos analisados foram identificados como pertencendo ao gênero Ocotea, da família Lauraceae, plantas vernaculamente conhecidas como canela. Os fragmentos são muito provavelmente provenientes de um único indivíduo, sendo que vários deles apresentam características de um nó, o que pode estar relacionado a aspectos tafonômicos de preservação diferencial.

As características anatômicas comumente encontradas em Lauraceae são: porosidade difusa; poros solitários e múltiplos, freqüentemente em cadeias; placas de perfuração simples, raramente escalariformes; parênquima paratraqueal escasso ou vasicêntrico, ocasionalmente aliforme, com bandas terminais em alguns gêneros; raios geralmente 2-3 seriados, homogêneos a fracamente heterogêneos, geralmente com presença de células oleiferas (Metcalfe \& Chalk 1950).

Os fragmentos analisados apresentaram as seguintes características: anéis de crescimento ausentes ou indistintos; poros difusos, dispersos, 
solitários e múltiplos de 2 a 4, com diâmetro tangencial de $50-100 \mu \mathrm{m} ; 30$ a 50 poros por $\mathrm{mm}^{2}$; tiloses freqüentes; placas de perfuração simples; pontoações intervasculares alternas, medindo 8-12 $\mu \mathrm{m}$; pontoações radiovasculares alongadas horizontalmente, maiores que as intervasculares; parênquima paratraqueal vasicêntrico; raios (2)3-4 seriados, 11 por $\mathrm{mm}$, subhomogêneos (células procumbentes com uma fileira marginal de células eretas); numerosas células oleiferas associadas aos raios e ao parênquima axial (Fig. 2).

A família das Lauraceae inclui cerca de 2500 espécies agrupadas em cerca de 50 gêneros, dos quais 25 ocorrem no Brasil. Destaca-se na composição florística de diversas fitofisionomias nativas, em especial as da Região Sul (floresta ombrófila densa, floresta ombrófila mista e, com menor diversidade, floresta aluvial e restinga). Geralmente são árvores e arbustos aromáticos, estando entre as principais espécies consideradas como produtoras de madeira de lei (Souza \& Lorenzi 2005).

O gênero Ocotea, com ampla distribuição desde o México e sul da Flórida até a Argentina, é o que apresenta o maior número de espécies nos Neotrópicos (cerca de 300). No Brasil, estima-se que existam entre 120 e 160 espécies (Baitello 2001).

A reduzida variabilidade de caracteres morfo-anatômicos entre as diversas espécies contribui para que esta família seja, de modo geral, considerada de difícil identificação. Somado a isso, a carência de estudos e conseqüentemente de descrições sobre a anatomia da madeira para grande parte das espécies restringe a identificação taxonômica ao nível de gênero (Werff 1991).

\section{Discussão}

Os fragmentos analisados no sítio Jabuticabeira-II permitem inferir que estes constituíam uma estaca associada à estrutura funerária, a qual provavelmente foi queimada acidentalmente. A canela, madeira utilizada nesta estaca, possui propriedades específicas de resistência e durabilidade, aspectos que provavelmente foram levados em consideração na escolha deste espécime.

Diversos indicadores têm demonstrado a existência de uma intensa preocupação e "cuidado com o corpo" na cultura sambaquieira (Klökler 2001; Gaspar 2000; Fish 2000; Gaspar et al. 2007). Isto é indicado pelos acompanhamentos funerários, pela disposição das camadas funerárias, geralmente evitando a interposição dos corpos no solo, e pelo próprio processo construtivo e proporções monumentais do sítio (Gaspar 2000). Em vista disso, pode-se levantar a hipótese de que haveria um investimento significativo na
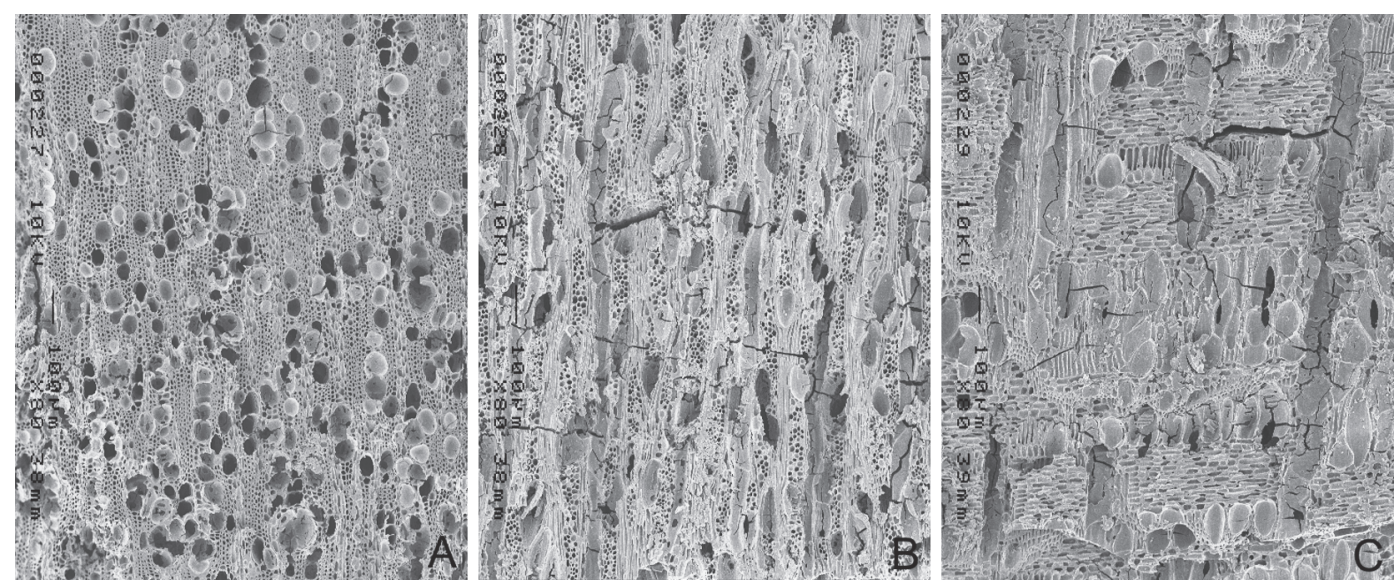

Fig. 2. Ocotea sp (Lauraceae). Micrografias em microscopia eletrônica de varredura de um fragmento de estaca carbonizada. A. Plano transversal. B. Plano longitudinal tangencial. C. Plano longitudinal radial. 
confecção da estrutura funerária, em termos de seleção das madeiras que a compõem e de construção da mesma. Considerando que o ritual funerário foi um aspecto central da cultura sambaquieira, é de se esperar que todo o material que integrava a cerimônia tivesse sido escolhido criteriosamente, integrando elementos cujas características fossem consideradas oportunas de acordo com o universo simbólico do grupo.

A seleção de uma madeira orientada pela necessidade de garantir durabilidade e resistência corrobora a idéia de zelo. A importância desta estrutura tanto pode estar relacionada à necessidade de permanência, atuando como um marco espacial do sepultamento, quanto com um elemento de sustentação que poderia estar relacionado à prática de prováveis oferendas.

Além disso, considerando que os rituais mortuários foram provavelmente cuidadosamente elaborados, a utilização de uma madeira que produz essências agradáveis pode ser significativa. As evidências arqueológicas indicam uma grande proximidade entre as estacas e as fogueiras, de modo que elas estavam sujeitas à ação do calor, o que provocaria a volatilização de óleos essenciais.

Por outro lado, a coleta desta madeira deve ter exigido um certo investimento. A caminhada envolvendo a busca de uma espécie/espécimen adequada(o), a derrubada da árvore ou ramo e seu transporte são atividades que exigiriam força física, resistência e habilidade em lidar com os instrumentos, sugerindo uma atividade relacionada com o sexo masculino.

A identificação de uma dada espécie dentro da mata exige um conhecimento profundo dos recursos vegetais, além de uma estreita relação com o ambiente. Esta atividade pode ser atribuição de membros do grupo dotados de um conhecimento diferenciado, que poderiam ser os indivíduos responsáveis pela coleta ou outros, que acompanhariam a expedição para indicar as árvores a serem utilizadas. Em outros casos, este saber pode ser amplamente difundido na comunidade, sendo a identificação acessível a grande parte dos indivíduos do grupo.

Espécies de Ocotea ocorrem nas formações litorâneas, mas são muito comuns nas matas de encosta. Na hipótese de a madeira utilizada corresponder a um taxon florestal, isso implicaria num grande deslocamento de membros do grupo sambaquieiro para obtenção deste material e numa grande familiaridade do grupo, ou ao menos de alguns membros detentores de conhecimentos diferenciados, com as áreas de encosta da região.

\section{Conclusão}

Este artigo aborda a utilização de recursos vegetais dentro de uma perspectiva inovadora, sublinhando sua relevância não somente como fonte de subsistência e produção de artefatos, mas também e especialmente no que se refere aos aspectos simbólicos que regem as escolhas de grupos culturais.

Esta abordagem paleoetnobotânica poderá trazer à luz uma série de informações importantes sobre o comportamento sociocultural dos grupos sambaquieiros, abrangendo aspectos como área de captação de recursos, coleta generizada, seleção de espécies de acordo com estratificações sociais dentro do grupo, entre outros.

Porém, um maior investimento na análise de material botânico é necessário para que seja possivel avançar no conhecimento destas relações. Para isso, é imprescindível um maior cuidado no resgate destes vestígios, que muitas vezes passam despercebidos no contexto arqueológico mas que são portadores de uma insuspeitada diversidade de informações. 
BIANCHINI, G.F.; SCHEEL-YBERT, R.; GASPAR, M.D. Lauraceae post in funerary context (Jabuticabeira-II site, Santa Catarina, Brazil). Revista do Museu de Arqueologia e Etnologia, São Paulo, 17: 223-229, 2007.

Abstract: Anthracology contributes with important information about use of plant resources by sambaqui moundbuilders. Analysis of a charred post associated to a funerary feature from site Jabuticabeira-II identified a Lauraceae (Ocotea sp.) and allowed to hypothesize about selective plant gathering. This selection might be driven by functional aspects of the wood characters and/or by symbolic aspects related to the cosmological domain of this group.

Keywords: Anthracology - Palaeoethnobotany - Shellmounds - Mortuary ritual.

\section{Referências bibliográficas}

BAITELLO, J. B.

2001 Novas espécies de Lauraceae para a flora brasileira. Acta Botanica Brasilica, 15 (3): 445-450.

DIAS JR, O.F.

1980 Rio de Janeiro: a tradição Itaipu e os sambaquis. P.I. SCHMITZ (Ed.) Anuário de Divulgação Científica do Instituto Goiano de Pré-História. Temas de Arqueologia Brasileira, 3: 33-34.

FISH, S.; DEBLASIS, P.; GASPAR, M. D.; FISH, P. 2000 Eventos incrementais na construção de sambaquis, litoral sul do Estado de Santa Catarina. Revista do Museu de Arqueologia e Etnologia, São Paulo, 10: 69-87.

GASPAR, M.D.

2000 Sambaqui: arqueologia do litoral brasileiro. Rio de Janeiro: Jorge Zahar Editor. 89 p.

GASPAR, M.D.; BUARQUE, A.; CORDEIRO, J.; ESCÓRCIO, E.

2007 Tratamento dos Mortos entre os Sambaquieiros, Tupinambá e Goitacá que ocuparam a Região dos Lagos, Estado do Rio de Janeiro. Revista do Museu de Arqueologia e Etnologia, São Paulo, 17: 169-189.

HANAZAKI, N.

2001 Ecologia de caiçaras: uso de recursos e dieta. Tese de Doutorado. Campinas, Universidade Estadual de Campinas, Instituto de Biologia. 193 p.

IAWA COMMITTEE.

1989 IAWA list of microscopic features for hardwood identification. E.A.Wheeler; P. Baas and P.E.Gasson (Eds.) IAWA Bulletin, n.s., 10 (3): 219-332.

KLÖKLER, D.M.

2001 Construindo ou deixando um Sambaqui? Análise de sedimentos de um sambaqui do litoral meridional brasileiro: processos formativos, região de Laguna, SC. Dissertação de Mestrado. São Paulo, FFLCHUSP. 164 p.

METCALFE, C; CHALK, L.

1950 Anatomy of the Dicotyledons. Oxford: Claredon Press. 1500 p.

PRANCE, G.

2005 The seeds of time. G. Prance and M. Nesbit (Eds.) The cultural history of plants. New York: Routledge. 452 p.

PROUS, A.

1992 Arqueologia Brasileira. Brasília: UNB. 620 p. SCHEEL-YBERT, R.

1999 Paleoambiente e paleoetnologia de populações sambaquieiras do sudeste do Estado do Rio de Janeiro. Revista do Museu de Arqueologia e Etnologia, São Paulo, 9: 43-59.

2001 Man and vegetation in the Southeastern Brazil during the Late Holocene. Journal of Archaeological Science 28 (5): 471-480.

2003 Relações dos habitantes de sambaquis com o meio ambiente: evidências de manejo de vegetais na costa sul-sudeste do Brasil durante o Holoceno Superior. IX Congresso 
da Associação Brasileira de Estudos do Quaternário. Recife: Video Congress. 5 p.

2004 Teoria e métodos em antracologia. 2. Técnicas de campo e de laboratório. Arquivos do Museu Nacional, Rio de Janeiro, 62 (4): 343-356.

SCHEEL-YBERT, R.; EGGERS, S.; WESOLOWSKI, V.; PETRONILHO, C.C.; BOYADJIAN, C.H.; DE BLASIS, P.A.D.; BARBOSA-GUIMARÃES, M.; GASPAR, M.D.

2003 Novas perspectivas na reconstituição do modo de vida dos sambaquieiros: uma abordagem multidisciplinar. Revista de Arqueologia, São Paulo, 16: 109-137.

SCHEEL-YBERT, R.; KLÖKLER, D.; GASPAR, M.D.; FIGUTI, L.

2005 Proposta de amostragem padronizada para macro-vestígios bioarqueológicos: antracologia, arqueobotânica, zooarqueologia. Revista do Museu de Arqueologia e Etnologia, São Paulo, 15. 16:139-163.

SCHEEL-YBERT, R.; CARVALHO, M.A.; MOURA, R.P.O.; GONÇALVES, T.A.P.; SCHEEL, M.; YBERT, J.P.

2006 Coleções de referência e bancos de dados de estruturas vegetais: subsídios para estudos paleoecológicos e paleoetnobotânicos. Arquivos do Museu Nacional, Rio de Janeiro, 64 (3): 255-266.

SCHMITZ, P.I.

2006 O mundo da caça, da pesca e da coleta. P.I. Schmitz (Ed.) Arqueologia do Rio Grande do Sul, Brasil. Documentos n 05. São Leopoldo: Instituto Anchietano de Pesquisas.

SIMÕES, C.B.

2007 O processo de formação dos sambaquis: uma leitura estratigráfica do sítio Jabuticabeira II, SC. Dissertação de Mestrado. São Paulo, MAE-USP. 248 p.

SOUZA, V.C.; LORENZI, H

2005 Botânica Sistemática: guia ilustrado para identificação das famílias de Angiospermas da flora brasileira, baseado em APGII. Nova Odessa: Instituto Plantarum. 640 p.

WERFF, H. VAN DER.

1991 A key to the genera of Lauraceae in the New World. Annals of the Missouri Botanical Garden, 78 (2): 377-387. 